\title{
Mobile vegetations on a poorly endothelialized atrial septal defect closure device
}

\author{
Hideki Kitamura, MD, PhD, ${ }^{\mathrm{a}}$ Masanori Yamamoto, MD, ${ }^{\mathrm{b}}$ and Ai Kagase, MD, ${ }^{\mathrm{b}}$ Nagoya, Japan
}

\footnotetext{
From the Departments of ${ }^{\mathrm{a} C}$ Cardiovascular Surgery and ${ }^{\mathrm{b}}$ Cardiology, Nagoya Heart Center, Nagoya, Japan. Disclosures: The authors reported no conflicts of interest.

The Journal policy requires editors and reviewers to disclose conflicts of interest and to decline handling or reviewing manuscripts for which they may have a conflict of interest. The editors and reviewers of this article have no conflicts of interest.

Received for publication Jan 9, 2021; accepted for publication Jan 11, 2021; available ahead of print Jan 13, 2021. Address for reprints: Hideki Kitamura, MD, PhD, Department of Cardiovascular Surgery, Nagoya Heart Center, 1-1-14 Sunadabashi, Higashi-ku, Nagoya, Aichi 461-0045, Japan (E-mail: kitamura@ heart-center.or.jp). JTCVS Techniques 2021;7:240-1

2666-2507

Copyright (c) 2021 The Authors. Published by Elsevier Inc. on behalf of The American Association for Thoracic Surgery. This is an open access article under the CC BY-NC-ND license (http://creativecommons.org/licenses/bync-nd/4.0/).

https://doi.org/10.1016/j.xjtc.2021.01.010
}

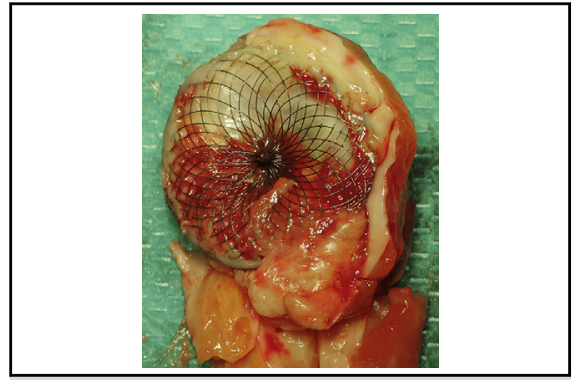

Giant mobile vegetations on a poorly endothelialized atrial septal defect closure device.
A 20-year-old man with atopic dermatitis was transferred to our hospital for urgent surgical treatment for infective endocarditis. The diagnosis was confirmed by blood cultures identifying methicillin-sensitive Staphylococcus aureus. Three years before this hospitalization, he had undergone percutaneous atrial septal defect closure using an Amplatzer septal occluder device (Abbott, St Paul, Minn). Transesophageal echocardiography showed a giant mobile mass suspected to represent vegetations around the device at the left atrial site without residual shunt (Figure 1, $A-C$, and Video 1). Through a median sternotomy, cardiopulmonary bypass was established with ascending aortic and bicaval cannulations. After cardioplegic arrest, the right atrium was opened and giant vegetations were observed not only at the left atrial site, but also at the right atrial site (Video 1). The device was removed along with the giant vegetations. Radical debridement resulted in large defects in both left and right atrial walls. The left atrial wall was reconstructed with a bovine pericardial patch. A second pericardial patch was then used to reconstruct the right atrial site defect, including interatrial septum. The removed vegetations had mainly proliferated in a poorly endothelialized area of the atrial septal defect closure device. (Figure 1, D).

The patient's postoperative course was uneventful and intravenous levofloxacin therapy was continued for 4 weeks. The patient was then discharged home in good condition,

\section{CENTRAL MESSAGE \\ Giant mobile vegetations around an atrial septal defect closure device were found on transeso- phageal echocardiography 3 years after implantation, prolif- erating mainly in poorly endo- thelialized areas.}

See Commentaries on pages 242 and 244. with ongoing oral levofloxacin antibiotic therapy. Anticoagulation therapy with warfarin was continued for 3 months postoperatively.

Currently, antiplatelet monotherapy and prophylaxis against infectious endocarditis is recommended to be continued for 6 months after atrial septal defect closure device implantation. Animal experiments have revealed mature endothelialization of the atrial septal defect closure device within 3 months. The risk of infectious endocarditis is believed to be decreased in the late phase after device implantation. However, intraoperative findings in this case demonstrated poor endothelialization of the device even 3 years after implantation. In addition, giant mobile vegetations had proliferated at both atrial sites, risking catastrophic systemic embolization. Although rare, cases of device endocarditis in the late phase up to 12 years have been reported. ${ }^{1-3}$ Several articles have demonstrated poor endothelialization after atrial septal defect closure device implantation. ${ }^{1-3}$ These findings indicate the continued risks of thrombotic and infectious complications over the long-term in some patients after 


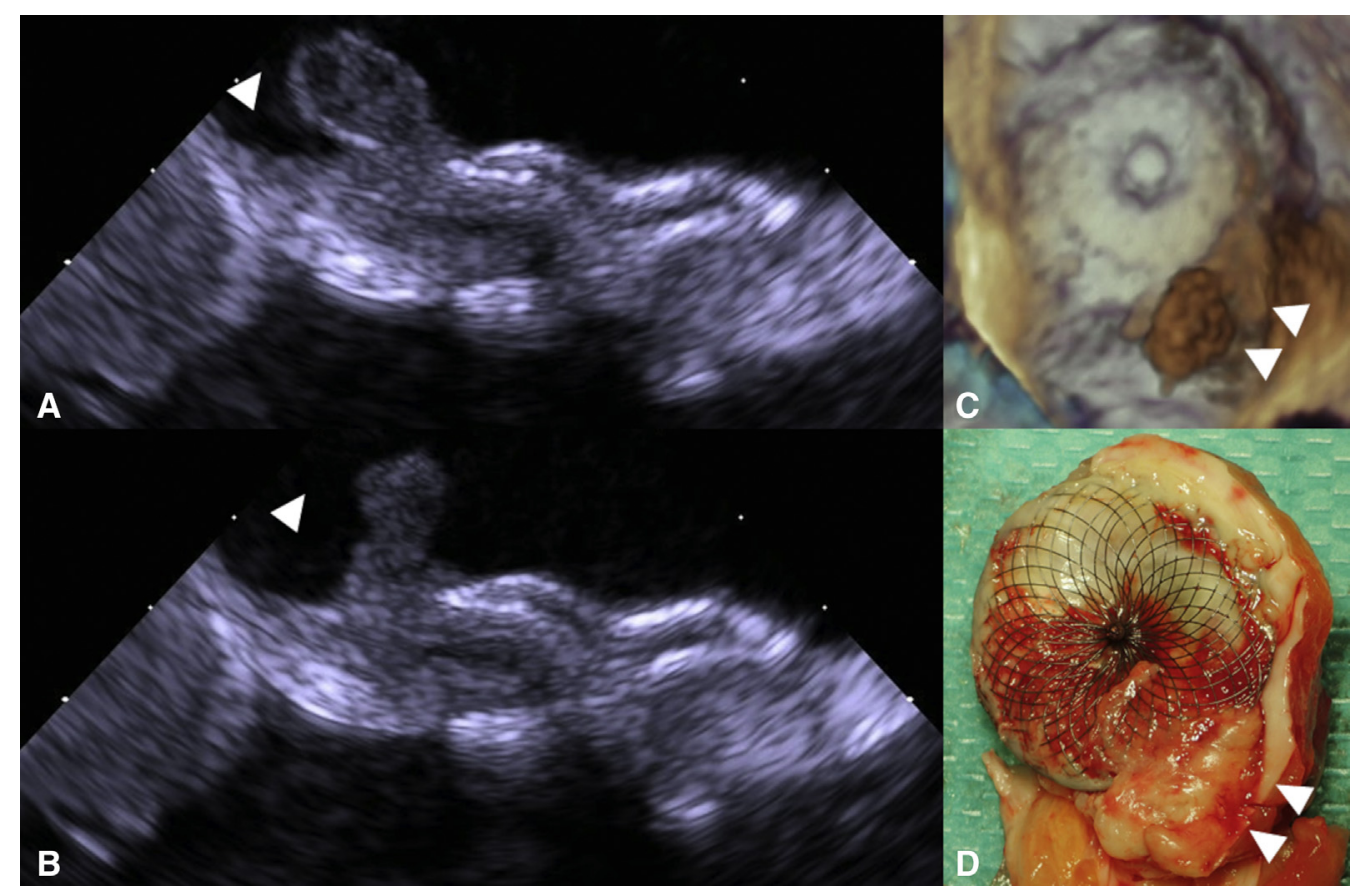

FIGURE 1. A and B, Transesophageal echocardiography shows a giant mobile mass (arrowhead) suspected to represent vegetation around the atrial septal defect closure device at the left atrial site. C, Three-dimensional image of the atrial septal defect closure device and mobile vegetations (arrowheads). D, Removed device and vegetations. Giant mobile vegetations (arrowheads) had proliferated mainly in poorly endothelialized areas.

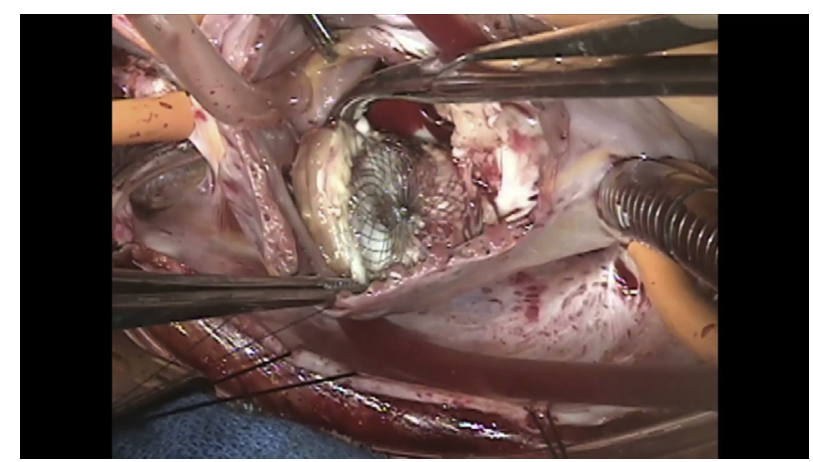

VIDEO 1. Findings of giant mobile vegetations on the atrial septal defect closure device. Video available at: https://www.jtcvs.org/article/S26662507(21)00064-X/fulltext.

implantation of an atrial septal defect closure device. Because atopic dermatitis is known as a risk factor for infectious endocarditis, ${ }^{4}$ surgical atrial septal closure could offer a good alternative to percutaneous device closure for patients with atopic dermatitis.
This study was approved by the Nagoya Heart Center Ethics Committee on September 29, 2020 (approval no: NHC2020-2929-10), and written informed consent for publication of this report was obtained from the patient. This work was not supported by any specific grants from any funding agencies in the public, commercial, or not-forprofit section.

\section{References}

1. Slesnick TC, Nugent AW, Fraser CD Jr, Cannon BC. Incomplete endothelialization and late development of acute bacterial endocarditis after implantation of an Amplatzer septal occluder device. Circulation. 2008; 117:e326-7.

2. Nguyen AK, Palafox BA, Starr JP, Gates RN, Berdjis F. Endocarditis and incomplete endothelialization 12 years after Amplatzer septal occlude deployment. Tex Heart Inst J. 2016;43:227-31.

3. Jha NK, Kiraly L, Murala JS, Tamas C, Talo H, EI Badaoui H, et al. Late endocarditis of Amplatzer atrial septal occluder device in a child. World J Cardiol. 2015;7: 703-6.

4. Onoda K, Mizutan H, Komada T, Kanemitsu S, Shimono T, Shimpo H, et al. Atopic dermatitis as a risk factor for acute native valve endocarditis. J Heart Valve Dis. 2000;9:469-71. 